\title{
Pre-trained deep learning models in automatic COVID-19 diagnosis
}

\author{
Ahmed Wasif Reza, Md Mahamudul Hasan, Nazla Nowrin, Mir Moynuddin Ahmed Shibly \\ Department of Computer Science and Engineering, East West University, Dhaka, Bangladesh
}

\begin{tabular}{l} 
Article Info \\
\hline Article history: \\
Received Feb 2, 2021 \\
Revised Mar 21, 2021 \\
Accepted Mar 29, 2021 \\
\hline Keywords: \\
Biomedical image classification \\
COVID-19 \\
Deep learning \\
EfficientNetB4 \\
Transfer learning
\end{tabular}

\begin{abstract}
Coronavirus disease (COVID-19) is a devastating pandemic in the history of mankind. It is a highly contagious flu that can spread from human to human without revealing any symptoms. For being so contagious, detecting patients with it and isolating them has become the primary concern for healthcare professionals. This study presented an alternative way to identify COVID-19 patients by doing an automatic examination of chest X-rays of the patients. To develop such an efficient system, six pre-trained deep learning models were used. Those models were: VGG16, InceptionV3, Xception, DenseNet201, InceptionResNetV2, and EfficientNetB4. Those models were developed on two open-source datasets that have chest X-rays of patients diagnosed with COVID-19. Among the models, EfficientNetB4 achieved better performances on both datasets with $96 \%$ and $97 \%$ of accuracies. The empirical results were also exemplary. This type of automated system can help us fight this dangerous virus outbreak.
\end{abstract}

This is an open access article under the $\underline{C C B Y-S A}$ license.

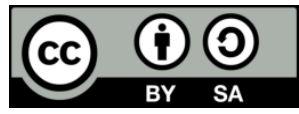

\section{Corresponding Author:}

Ahmed Wasif Reza

Department of Computer Science and Engineering

East West University

Dhaka, Bangladesh

Email: wasif@ewubd.edu

\section{INTRODUCTION}

Coronavirus disease (COVID-19) has become a worldwide outbreak and is an ongoing pandemic. It can transmit from human to human without revealing any symptoms. The COVID-19's epidemiological features are still a matter of scrutiny. The statistics so far indicate that about $80 \%$ of COVID-19 patients are not in more than mild conditions and about $20 \%$ are in severe conditions. Besides, approximately $10 \%$ of patients need mechanical ventilators. The rate of death is about $2 \%$, although researchers consider that the death rate is $0.5 \%$ lower [1]. To treat the Covid-affected patient properly, it is vital to seek out whether a patient is being affected. A standard polymerase chain reaction (PCR) test can be performed on the samples of the patients, but it can be costly and time-consuming. An alternative to the standard COVID-19 detection tests can be identifying the affected patients from chest X-rays. The COVID-19 patients who are more vulnerable to casualty are those whose lungs are affected by the virus. Therefore, examining chest X-rays can be a very appropriate method to make a prediction about a patient that has COVID-19 or not. However, only specialist physicians can diagnose COVID-19 by examining the chest X-ray. There is a scarcity of doctors who can perform such a diagnosis. In these circumstances, an automated and intelligent predictive system that can diagnose patients with COVID-19 can be very helpful. Such an automated detection mechanism can reduce the stress of the healthcare system in a meaningful way by identifying the affected patients rapidly and accurately. 
This study has aimed at developing appropriate predictive models that can perform diagnosis on the chest X-ray images of suspected patients. The deep learning models have been utilized to create those predictive models. To develop such predictive models with high efficiency, a large dataset is needed. However, there are a very limited number of chest X-ray images of COVID-19 affected patients are available publicly. Acquiring a large number of appropriate images is also not feasible. In this situation, the concept of transfer learning can come to aid. There are a handful of deep learning models that are pre-trained on large datasets. Their acquired knowledge can be transferred to solve the task of COVID-19 detection with small datasets. Since they already know so many aspects of various images, those models can slightly adjust their weights based on the X-ray images and make decent predictions. This study has explored the applicability of pre-trained deep learning models in the COVID-19 detection task. To achieve this goal, six popular pretrained deep learning models have been trained specially for the COVID-19 recognition task. After that, a comparative analysis of the models has been performed. The major contributions of this study are: i) Developing automatic diagnostic system based on pre-trained deep learning models for COVID-19 detection from chest X-ray images; ii) Achieving better performance than existing works in COVID-19 detection task using EfficientNetB4 pre-trained model; iii) In the following section of this article, the related works of this study are presented. Then, the methods and materials of the various experiments have been described followed by the results of this study with appropriate discussion. In the last section of this article, few concluding remarks have been mentioned.

\section{RELATED WORKS}

Majeed et al. [2] have provided an analysis of 12 regular convolutional neural network models to help radiologists discriminate against COVID-19 based on chest X-rays, and also have introduced a CNN model that could give efficient final prediction results. The proposed model has been designed to perform reliable diagnostics for COVID vs. Normal classification and COVID vs. Normal vs. Pneumonia classification. Their classifier has provided $98.08 \%$ accuracy for binary classification and $87.02 \%$ accuracy for multiclass classification. The DarkNet has been proposed in this study [3] as a classifier for COVID-19 diagnosis. In another study [4], the experimental findings have shown the ability of the DeTraC (Decompose, Transfer, and Compose) model to identify COVID-19 cases from a large data collection of images obtained from hospitals around the world. High accuracy has been obtained by DeTraC for the identification of COVID-19 from chest X-rays. Cohen et al. [5] have gathered medical images from websites and publications, and the dataset currently contains 123 front-view X-rays of COVID-19 patients. The authors of few works [6], [7] have used pre-trained deep learning classifiers for COVID-19 diagnosis. In another work, it has been proved that the automated system can detect pneumonia from chest X-rays with higher accuracy in less time than human experts [8]. The authors of these works [9]-[14] have used various deep learning methods for the automatic COVID-19 diagnosis task. Another article [15] has introduced a framework focused on Capsule Networks which has been named COVID-CAPS, which can manage small datasets that are of major significance. COVID-CAPS has reached an accuracy of $95.7 \%$ with a much smaller number of training parameters. A faster R-CNN model has also been introduced to detect COVID-19 patients from chest X-ray images [16]. In Table 1 (see appendix), a summary of few state-of-the-art automated works on COVID-19 diagnosis has been presented.

\section{METHODS AND MATERIALS}

This study aims at detecting COVID-19 from biomedical images so that early and appropriate treatment of the affected patients is possible. The overall working procedure of this study is presented in Figure 1. In this section of the article, the methods and the materials of this study are presented. First, the dataset that has been used is described. After that, the methods of identifying COVID-19 from X-ray images are presented.

\subsection{Dataset}

There are several publicly available datasets of COVID-19 X-ray images. A COVID-19 X-ray image database has been developed by Cohen et al. [5] and another one is available at the Kaggle repository [21]. After collecting the X-ray images from these two sources, both datasets are combined and refined using domain knowledge. Both datasets have three classes of COVID-19, Normal, and Pneumonia. Dataset-1 contains a total of 1,780 X-ray images with 460 images of COVID-19 infected, 662 normal, and 665 Pneumonia. Dataset-2 contains a total of 2,990 X-ray images, including 990 COVID-19-infected images, 1,000 Normal and 1,000 Pneumonia images. 


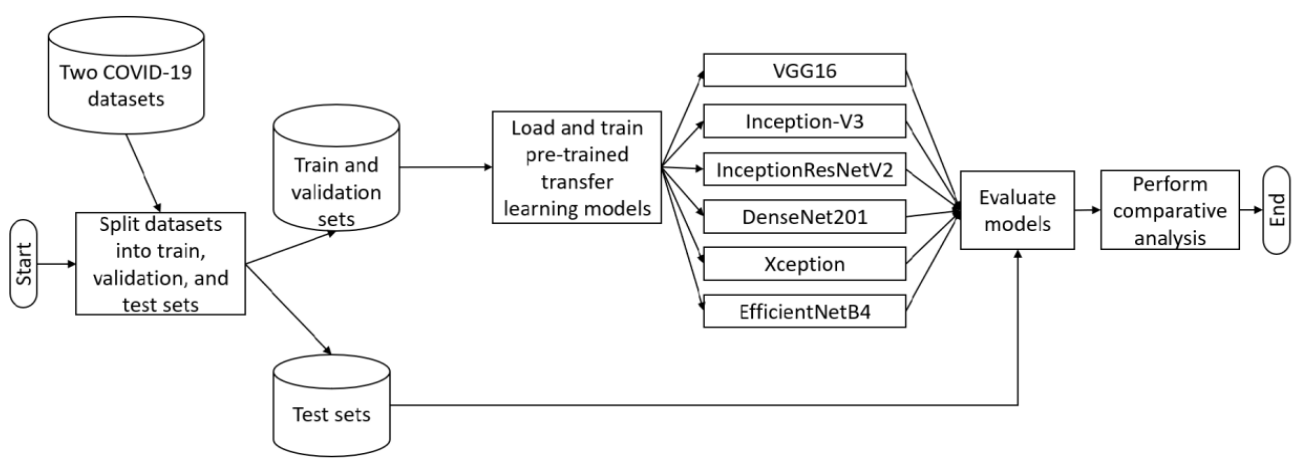

Figure 1. Proposed methods of this study

\subsection{Data pre-processing and image augmentation}

To train a machine learning classifier, the pixel-value representation of images is required. The pixel values of an image are between 0 and 255. The pixel value-wise representation of the images is rescaled between 0 and 1 so that the machine learning model can work properly on the inputs. We have used this normalization function in both test and training datasets. The images captured by a camera have various sizes. But the deep learning architecture should be provided with fixed-sized images. Therefore, we have resized all the images to $299 \times 299$ before training.

Image augmentation is a powerful method in building convolutional neural networks that can increase the size of the training set without acquiring new images. As part of the data augmentation, we have rotated, cropped, and flipped the images to fit the model as needed. By flipping the image horizontally, vertically, and rotating we can completely rearrange the pixels. The Keras deep learning neural network library provides the capability to fit models using image data augmentation via the ImageDataGenerator class. Image data augmentation is used to expand the training dataset to improve the performance and ability of the model to generalize.

\subsection{Pre-trained models}

The biggest problem in the analysis of medical data is the lack of adequate datasets. The deep learning classifiers need a large amount of data to be trained on. Collecting more data for the domain-specific tasks and annotating them is costly and time-consuming. To overcome the limitation of having a large dataset in the task of COVID-19 detection, various deep transfer learning models have been employed in this study. The biggest advantage of using the transfer learning method is that it can train data with smaller datasets and requires less training time. Transfer learning is regularly used in deep learning and the wisdom obtained by the pre-trained model is conveyed to the classifier to be trained. Six pre-trained models have been used to detect COVID-19 from X-rays and those are VGG16, DenseNet201, InceptionV3, InceptionResNetV2, Xception, and EfficientNet. Those models have been pre-trained on the ImageNet dataset that has 1000 classes of images. For being trained on such a huge dataset, the learned weights of those models can be leveraged to classify the images of the biomedical domain.

The first architecture that has been used for COVID-19 detection is VGG16. It is a model of the deep neural network. This network has been introduced by Simonyan and Zisserman [22]. VGG16 starts with two convolutional layers. After that, there is a max-pooling layer in the network. It is followed by two convolutional layers and a max-pooling layer. After that, there are three convolutional layers followed by a pooling layer in the network. Again, there are three convolutional layers in the architecture followed by a max-pooling layer which repeats two times. The architecture ends with three dense layers. The last of these dense layers is the output layer.

Another architecture that has been used is InceptionV3 [23] which is an extended network of GoogleNet. It has achieved strong classification efficiency using transfer learning in several biomedical applications. The Xception [24] architecture is a linear stack of depthwise separable convolution layers with residual connections which imply that spatial convolution and cross channel convolution is completely separated. Following GoogLeNet, the InceptionV3 model has been proposed to concatenate several convolutional filters of various sizes into a new filter. Such a design minimizes the number of parameters to be learned, and the computational complexity is thereby reduced. It is a 48-layer large convolutional neural network. InceptionV3 is a convolutional neural network design of the Inception family. Xception architecture focused entirely on separable convolutional layers in depth. Xception network has been build based on the Inception network. In Xception, the convolutional layers of inception blocks of the Inception network have 
been replaced with separable convolutional layers that give the same functionalities as convolutional layers but with less computation and with more efficiency. It is an efficient architecture that depends on depthwise separable convolution and shortcuts between convolution blocks as in ResNet.

DenseNet201 [25] is a deep convolutional neural network that is 201 layers deep. DenseNet contains few dense blocks. Each dense block has few convolutional layers. Within a dense block, the convolutional layers are connected to each previous layer. The dense blocks are also connected to each previous dense block. A deeper dense block not only processes the information received from the previous layers but also considers the outputs of all previous blocks. DenseNets have some persuasive advantages: they mitigate the vanishing gradient issue, promote the propagation of features, facilitate the reuse of features, and significantly minimize the number of parameters. The fifth deep learning architecture that has been employed in this work is InceptionResNetV2 [26]. It is a convolutional neural architecture that builds on the architecture family of Inception but integrates residual connections, replacing the Inception architecture's filter concatenation level. It has 164 layers. This network has been developed based on the Inception and the residual connection. Multiple-sized convolutional filters are combined with residual connections in the Inception-Resnet block.

Each of the CNN architectures has some unique characteristics. EfficientNets are a family of image classification models that achieved state-of-the-art accuracy yet being an order-of-magnitude smaller and faster than other deep learning models [27]. The core idea of EfficientNet architecture is about strategically scaling deep neural networks. The scaling method introduced in EfficientNets is named compound scaling and suggests that instead of scaling only one model attribute out of depth, width, and resolution; strategically scaling all three of them together delivers better results. Model scaling is about scaling the existing model in terms of model depth, model width, and less popular input image resolution to improve the performance of the model. Scaling up any dimension of network width, depth, or resolution improves accuracy, but the accuracy gain diminishes for bigger models. It is critical to balance all dimensions of network width, depth, and resolution during scaling. EfficientNet is one of the most efficient architectures for image classification.

\section{EXPERIMENTAL SETUP}

This work aims at detecting COVID-19 from x-rays. For developing the transfer learning models, Keras on top of TensorFlow-a python library has been utilized. The models have been trained on a computer having Intel Core i5 7200U CPU and 8 GB RAM, with Windows 10 operating system. The datasets have been split into three sets-train, validation, and test sets. The training, validation, and test sets have $75 \%, 10 \%$, and 15\% X-ray images, respectively. During training the models, after each epoch, the models have been validated with a validation set. For having three classes (Normal, Pneumonia, and COVID-19) in the datasets, the classification problem of this study is a multi-class classification problem. Therefore, a categorical cross-entropy loss function has been used. Additionally, adam optimizer has been used for all the models during training. After the completion of training, the models have been tested against the test set, and based on few evaluation metrics, the performance of the trained models is reported.

\subsection{Evaluation metrics}

The developed models have been evaluated using a multi-class confusion matrix. Precision, recall, f1-score, accuracy, and specificity have been used to evaluate the models. Precision is the portion of the correct predictions made by the classifier with respect to total predicted classes. The recall is the portion of the correct predictions made by the classifier regarding the total existing accurate classes. F1-score is the harmonic mean of precision and recall i.e., both precision and recall are given equal importance while evaluating the performance of a classifier. Accuracy is known as the overall recognition rate. And Specificity is the true negative rate. If $T P, T N$, $F P, F N, P$, and $N$ are true positive, true negative, false positive, false negative, the total number of positive instances, and the total number of negative instances, respectively, then

$$
\begin{aligned}
& \text { precision }=\frac{\mathrm{TP}}{\mathrm{TP}+\mathrm{FP}} \\
& \text { ecall }=\frac{\mathrm{TP}}{\mathrm{P}} \\
& \text { f1_score }=\frac{2 \times \text { precision } \times \text { recall }}{\text { precision+recall }} \\
& \text { accuracy }=\frac{\mathrm{TP}+\mathrm{TN}}{\mathrm{P}+\mathrm{N}} \\
& \text { specificity }=\frac{\mathrm{TN}}{\mathrm{N}}
\end{aligned}
$$




\section{RESULTS AND DISCUSSION}

The results of various experiments of this study have been outstanding. Better performing automated models that can detect a COVID-19 patient from an x-ray image can be very helpful to fight the ongoing pandemic. The six pre-trained deep learning models have demonstrated overall good results in the detection task. In this section of the article, the results of this study with various aspects are presented.

All the models have been trained for 20 epochs and their training and validation accuracies are observed to get to know whether the learning of the models has been appropriate or not. In Figure 2, a comparison of training accuracies of the six models for both datasets is presented. The comparison of validation accuracies is also given in the figure. From the figure, it is observed that for the first dataset, Xception and EffecientNetB4 models have demonstrated better accuracies than other models. The Xception has taken first place in terms of training accuracy while the EffecientNetB4 has taken the first place of validation accuracy. The EffecientNetB4 model has continued its better performance for the second dataset achieving first place for both training and validation accuracy while the InceptionV3 model has achieved second place in both evaluation metrics. The other models have shown good performances but not as good as the described ones. In Figure 3, the comparison of losses for the models is presented. Just like training and validation accuracies, EffecientNetB4, Xception, and InceptionV3 models have been the top performers in terms of training and validation losses. On the other hand, VGG16 and InceptionResNetV2 models have been worse in terms of training loss for both datasets. And for validation loss, DenseNet201, InceptionResNetV2, and VGG16 have been poor performers across the datasets.

Training time is an important aspect of training deep learning models. The reason for using the transfer learning models is to reduce the cost of training. The training time of the models used in this study has been observed. In Table 2, the average latency per step of the models on two datasets is given. From the table, it is apparent that VGG16, DenseNet201, and EffecientNetB4 have taken lesser time to train than other models. Xception has taken almost 15 seconds per step to train which is the slowest among the six pretrained models.

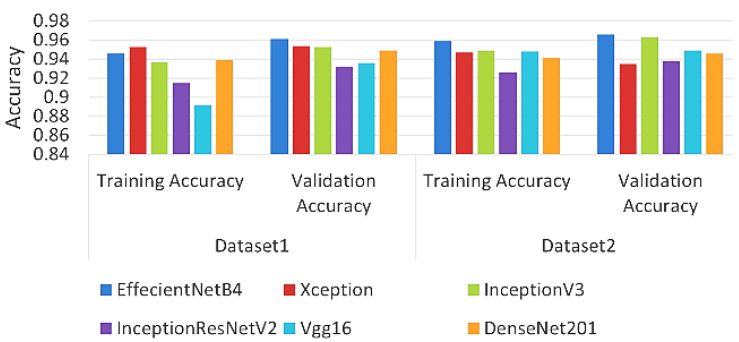

Figure 2. Training and validation accuracy comparison for two datasets

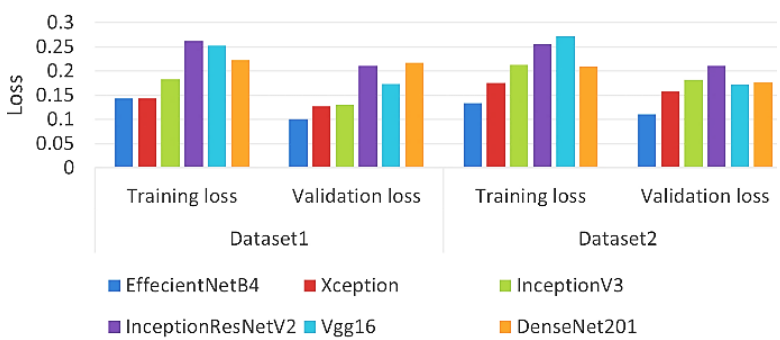

Figure 3. Training and validation loss comparison for two datasets

Table 2. Training time comparison of the deep learning models

\begin{tabular}{lc}
\hline \multicolumn{1}{c}{ Architecture } & Latency Per Step (millisecond) \\
\hline EffecientNetB4 & 658 \\
InceptionV3 & 9,000 \\
Vgg16 & 554 \\
InceptionResNetV2 & 8,000 \\
DenseNet201 & 599 \\
Xception & 15,000 \\
\hline
\end{tabular}

In Table 3, the class-wise performances of the models across two datasets are presented. Precision and recall of the classifiers have also been presented. Because just knowing the accuracies of the classifiers for the classification tasks is not good enough in the medical domains. In this case, identifying a COVID-19 patient precisely is more important than identifying a normal patient accurately. Therefore, along with accuracies, other evaluation metrics like precision, recall, and specificity are also needed to prove the applicability of a classifier in a real-world environment. Moreover, class-wise performances are presented in Table 3 to give more clarity of the models' appropriateness. From the table, it can be seen that when it comes to performing better on the test set for COVID-19 detection, the EffecientNetB4 model has achieved better performances for both datasets. It has achieved 0.96 and 0.97 accuracies for two datasets, respectively. Additionally, for the class COVID-19, it has demonstrated a 0.99 of f1-score on both test sets. The 
EffecientNetB4 has also yielded 0.99 of precision on the first dataset and 1.00 of precision on the second dataset. This model has also demonstrated the highest specificity of 0.95 on the first dataset and the highest specificity of 0.96 on the second dataset for the COVID-19 class. However, among the six models, for detecting COVID-19, the Xception model has the highest recall of 1.00 and 0.99 for the two datasets, respectively. From Table 3, it is also apparent that the other models except EffecientNetB4 have also demonstrated good performances. The overall good results also indicate the usability of such deep learning models in detecting COVID-19 patients from corresponding chest X-ray images.

Table 3. Class-wise performances of the deep learning models

\begin{tabular}{|c|c|c|c|c|c|c|c|}
\hline Dataset & Model & Label & Recall & Specificity & Precision & F1-Score & Accuracy \\
\hline \multirow[t]{18}{*}{ Dataset 1} & \multirow{3}{*}{ EffecientNetB4 } & COVID-19 & 0.99 & 0.95 & 0.99 & 0.99 & \multirow[t]{3}{*}{0.96} \\
\hline & & Normal & 1.00 & 0.95 & 0.91 & 0.95 & \\
\hline & & Pneumonia & 0.90 & 0.99 & 1.00 & 0.95 & \\
\hline & \multirow[t]{3}{*}{ InceptionV3 } & COVID-19 & 0.98 & 0.94 & 0.99 & 0.98 & \multirow[t]{3}{*}{0.95} \\
\hline & & Normal & 1.00 & 0.92 & 0.89 & 0.94 & \\
\hline & & Pneumonia & 0.88 & 0.98 & 0.99 & 0.93 & \\
\hline & \multirow{3}{*}{ Vgg16 } & COVID-19 & 0.99 & 0.92 & 0.95 & 0.97 & \multirow[t]{3}{*}{0.94} \\
\hline & & Normal & 0.91 & 0.96 & 0.95 & 0.93 & \\
\hline & & Pneumonia & 0.93 & 0.94 & 0.91 & 0.92 & \\
\hline & \multirow[t]{3}{*}{ InceptionResNetV2 } & COVID-19 & 0.94 & 0.93 & 0.99 & 0.96 & \multirow[t]{3}{*}{0.93} \\
\hline & & Normal & 0.95 & 0.92 & 0.91 & 0.93 & \\
\hline & & Pneumonia & 0.91 & 0.94 & 0.92 & 0.92 & \\
\hline & \multirow[t]{3}{*}{ DenseNet201 } & COVID-19 & 0.97 & 0.95 & 0.99 & 0.98 & \multirow[t]{3}{*}{0.95} \\
\hline & & Normal & 0.96 & 0.94 & 0.93 & 0.94 & \\
\hline & & Pneumonia & 0.92 & 0.96 & 0.94 & 0.93 & \\
\hline & \multirow[t]{3}{*}{ Xception } & COVID-19 & 1.00 & 0.95 & 0.94 & 0.97 & \multirow[t]{3}{*}{0.96} \\
\hline & & Normal & 0.98 & 0.95 & 0.96 & 0.97 & \\
\hline & & Pneumonia & 0.92 & 0.98 & 0.98 & 0.95 & \\
\hline \multirow[t]{18}{*}{ Dataset 2} & \multirow[t]{3}{*}{ EffecientNetB4 } & COVID-19 & 0.98 & 0.96 & 1.00 & 0.99 & \multirow[t]{3}{*}{0.97} \\
\hline & & Normal & 0.99 & 0.96 & 0.93 & 0.96 & \\
\hline & & Pneumonia & 0.94 & 0.98 & 0.97 & 0.96 & \\
\hline & \multirow[t]{3}{*}{ InceptionV3 } & COVID-19 & 0.99 & 0.95 & 0.99 & 0.99 & \multirow[t]{3}{*}{0.96} \\
\hline & & Normal & 0.98 & 0.94 & 0.91 & 0.95 & \\
\hline & & Pneumonia & 0.90 & 0.98 & 0.97 & 0.94 & \\
\hline & \multirow[t]{3}{*}{ Vgg16 } & COVID-19 & 0.99 & 0.93 & 0.97 & 0.98 & \multirow[t]{3}{*}{0.95} \\
\hline & & Normal & 0.92 & 0.96 & 0.94 & 0.93 & \\
\hline & & Pneumonia & 0.94 & 0.96 & 0.94 & 0.94 & \\
\hline & \multirow[t]{3}{*}{ InceptionResNetV2 } & COVID-19 & 0.96 & 0.93 & 0.99 & 0.98 & \multirow[t]{3}{*}{0.94} \\
\hline & & Normal & 0.95 & 0.94 & 0.90 & 0.92 & \\
\hline & & Pneumonia & 0.90 & 0.96 & 0.93 & 0.91 & \\
\hline & \multirow[t]{3}{*}{ DenseNet201 } & COVID-19 & 0.97 & 0.94 & 0.99 & 0.98 & \multirow[t]{3}{*}{0.95} \\
\hline & & Normal & 0.95 & 0.94 & 0.92 & 0.94 & \\
\hline & & Pneumonia & 0.92 & 0.96 & 0.92 & 0.92 & \\
\hline & \multirow{3}{*}{ Xception } & COVID-19 & 0.99 & 0.92 & 0.99 & 0.99 & \multirow{3}{*}{0.94} \\
\hline & & Normal & 0.98 & 0.92 & 0.86 & 0.93 & \\
\hline & & Pneumonia & 0.84 & 0.98 & 0.98 & 0.91 & \\
\hline
\end{tabular}

EffecientNetB4 has been proved to be the most outstanding performer in the task of automatic COVID-19 diagnosis from chest X-rays. The comparison with the performances of other deep learning models has also proved the superiority of EffecientNetB4 in this biomedical image classification. Using EffecientNetB4 for COVID-19 detection in this work is also one of the very first works to be reported. To the best of our knowledge, there has not been any reported work with EffecientNetB4 for COVID-19 detection. The main reason behind the better performance of this deep learning architecture is its scalability. This architecture has been introduced with the most appropriate network dimension which allows the model to learn the most efficient aspects of the images and to use those to classify the images efficiently.

Transfer learning has played a significant role in the good performances of this study. All the models that have been used in the experiments are pre-trained on the ImageNet dataset. Those learnings have helped our models to work better on the specific classification task. Image augmentation has also helped the models to achieve better performance. As the datasets used in this study are relatively smaller and COVID-19 related chest $\mathrm{x}$-ray images are scarce, various augmentation techniques applied to the existing images have led the models to achieve better generalizability. Better balanced individual class-wise test performances in various evaluation metrics indicate the robustness and sustainability of the models. However, due to a lack of computational resources, the models have not been trained for more than 20 epochs. The longer training phase could have improved the models' performances. Another limitation of this work is not to explore the various hyperparameters of the deep learning architectures. Tuning those hyperparameters would surely allow the models to perform better. 
This study has demonstrated exceptional performance in the automatic diagnosis of COVID-19 from biomedical images. In doing so, this work has also beaten few state-of-the-art works related to the domainspecific classification task. In Table 4, a comparison of this work with a few of the other works is given. The EffecientNetB4 method is the standout performer among the methods implemented in our work. On two datasets, this method has $96 \%$ and $97 \%$ test accuracy, respectively. These performances are superior in comparison to other works like $89.60 \%$ test accuracy achieved by Khan et al. [28], 95\% test accuracy achieved by Waheed [29] and 87.02\% test accuracy achieved by Majeed et al. [2].

Table 4. Test accuracy comparison with other works

\begin{tabular}{lll}
\hline \multicolumn{1}{c}{ Work } & \multicolumn{1}{c}{ Method } & Test accuracy \\
\hline Khan et al. [28] & CoroNet & $89.60 \%$ \\
Waheed [29] & CovidGAN & $95 \%$ \\
Abbas et al. [4] & A deep CNN & $92.5 \%$ \\
Afshar [15] & COVID-CAPS & $95.7 \%$ \\
Majeed et al. [2] & 12 off-the-shelf CNN architectures & $87.02 \%$ \\
Proposed work (on dataset 1) & EffecientNetB4 & $96 \%$ \\
Proposed work (on dataset 2) & EffecientNetB4 & $97 \%$ \\
\hline
\end{tabular}

\section{CONCLUSION}

An intelligent system that identifies COVID-19 patients from their chest X-rays can be very helpful to fight the ongoing pandemic. The efficiency of those intelligent systems is the highest concern. This study is an attempt to develop such efficient intelligent and data-driven systems. This study develops six transfer learning models for the detection task. The models have been proved to be very efficient in the COVID-19 identification task. Among the developed models, EffecientNetB4 has yielded the best performance. There are few limitations of this study like not tuning hyperparameters, training the models for fewer epochs, not using other pre-trained models and not using the ensemble methods for better performances. Those experiments can be conducted to improve the efficiency of the predictive systems. Moreover, the working mechanism of deep learning models is not understandable to the common stakeholders. Research can be conducted to enhance the explainability of deep learning models in biomedical domains. However, despite having those limitations, the automatic detection system developed in this study can surely help us to overcome this appalling situation and fight this coronavirus outbreak.

\section{APPENDIX}

Table 1. A summary of few works in automated COVID-19 diagnosis from chest X-rays

\begin{tabular}{|c|c|c|c|c|}
\hline Work & Method & Dataset & Finding & Limitation/Gap \\
\hline Majeed et al. [2] & $\mathrm{CNN}-\mathrm{X}$ architecture & $\begin{array}{l}\text { Composition of three } \\
\text { publicly available } \\
\text { datasets }\end{array}$ & $\begin{array}{l}\text { CNN-X works better } \\
\text { than Xception and } \\
\text { DenseNet }\end{array}$ & $\begin{array}{l}\text { Contains text and medical } \\
\text { device traces on chest X-ray } \\
\text { images }\end{array}$ \\
\hline $\begin{array}{l}\text { Makris and } \\
\text { Tserpes [17] }\end{array}$ & $\begin{array}{l}\text { Nine deep learning } \\
\text { architectures }\end{array}$ & $\begin{array}{l}\text { Paul Cohen et al. }[5] \\
\text { and Kaggle }\end{array}$ & $\begin{array}{l}\text { VGG16 and VGG19 } \\
\text { show the best results than } \\
\text { other models. }\end{array}$ & $\begin{array}{l}\text { Lack of data could be evaluated } \\
\text { with more architectures. }\end{array}$ \\
\hline Jaiswal et al. [18] & $\begin{array}{l}\text { Transfer learning } \\
\text { model } \\
\text { with DenseNet201 }\end{array}$ & Kaggle & $\begin{array}{l}\text { Maximum Accuracy of } \\
99.82 \%\end{array}$ & $\begin{array}{l}\text { Lack of sophisticated feature } \\
\text { extraction techniques. }\end{array}$ \\
\hline Narin et al. [19] & $\begin{array}{l}\text { ResNet50, ResNet101, } \\
\text { ResNet152, Inception- } \\
\text { V3 and } \\
\text { InceptionResNetV2 }\end{array}$ & $\begin{array}{l}\text { One dataset containing } \\
\text { COVID-19 X-rays and } \\
\text { two datasets of general } \\
\text { chest X-rays. }\end{array}$ & $\begin{array}{l}\text { ResNet50 yielded the } \\
\text { highest accuracy }\end{array}$ & $\begin{array}{l}\text { Features are not extracted using } \\
\text { image processing methods. }\end{array}$ \\
\hline Abbas et al. [4] & Deep CNN & Cohen et al. [5] & $\begin{array}{l}\text { ResNet } 18 \text { performed } \\
\text { better. }\end{array}$ & $\begin{array}{l}\text { Lack of using other deep } \\
\text { learning models. }\end{array}$ \\
\hline Ozturk et al. [3] & DarkNet & Cohen et al. [5] & $\begin{array}{l}\text { Binary-class achieved } \\
\text { better accuracy than } \\
\text { multi-class classification. }\end{array}$ & Scarcity of COVID-19 X-rays. \\
\hline Salih [20] & Modified AlexNet & A dataset from Kaggle & $\begin{array}{l}\text { Modified AlexNet } \\
\text { performed better than } \\
\text { original AlexNet }\end{array}$ & $\begin{array}{l}\text { Lack of Balanced dataset and } \\
\text { not applying the modifications } \\
\text { to other deep learning models. }\end{array}$ \\
\hline
\end{tabular}




\section{REFERENCES}

[1] R. M. Pereira, D. Bertolini, L. O. Teixeira, C. N. Silla, and Y. M. G. Costa, "COVID-19 identification in chest Xray images on flat and hierarchical classification scenarios," Comput. Methods Programs Biomed., 2020, doi: 10.1016/j.cmpb.2020.105532.

[2] T. Majeed, R. Rashid, D. Ali, and A. Asaad, "Issues associated with deploying CNN transfer learning to detect COVID-19 from chest X-rays," Phys. Eng. Sci. Med., 2020, doi: 10.1007/s13246-020-00934-8.

[3] T. Ozturk, M. Talo, E. A. Yildirim, U. B. Baloglu, O. Yildirim, and U. Rajendra Acharya, "Automated detection of COVID-19 cases using deep neural networks with X-ray images," Comput. Biol. Med., 2020 , doi: 10.1016/j.compbiomed.2020.103792.

[4] A. Abbas, M. M. Abdelsamea, and M. M. Gaber, "Classification of COVID-19 in chest X-ray images using DeTraC deep convolutional neural network," Appl. Intell., 2021, doi: 10.1007/s10489-020-01829-7.

[5] J. Paul Cohen, P. Morrison, and L. Dao, “COVID-19 Image Data Collection,” arXiv. 2020.

[6] I. D. Apostolopoulos and T. A. Mpesiana, "Covid-19: automatic detection from X-ray images utilizing transfer learning with convolutional neural networks," Phys. Eng. Sci. Med., 2020, doi: 10.1007/s13246-020-00865-4.

[7] T. Rahman, M. E. H. Chowdhury, A. Khandakar, and A. Khandakar, "Transfer learning with deep Convolutional Neural Network (CNN) for pneumonia detection using chest X-ray," Appl. Sci., 2020, doi: 10.3390/app10093233.

[8] H. Sharma, J. S. Jain, P. Bansal, and S. Gupta, "Feature extraction and classification of chest X-ray images using CNN to detect pneumonia," 2020, doi: 10.1109/Confluence47617.2020.9057809.

[9] X. Xu, et al., “A deep learning system to screen novel coronavirus disease 2019 pneumonia," Engineering, 2020, doi: 10.1016/j.eng.2020.04.010.

[10] O. Stephen, M. Sain, U. J. Maduh, and D. U. Jeong, "An efficient deep learning approach to pneumonia classification in healthcare," J. Healthc. Eng., 2019, doi: 10.1155/2019/4180949.

[11] G. Jain, D. Mittal, D. Thakur, and M. K. Mittal, “A deep learning approach to detect Covid-19 coronavirus with X-Ray images,” Biocybern. Biomed. Eng., 2020, doi: 10.1016/j.bbe.2020.08.008.

[12] M. Toğaçar, B. Ergen, and Z. Cömert, "COVID-19 detection using deep learning models to exploit Social Mimic Optimization and structured chest X-ray images using fuzzy color and stacking approaches," Comput. Biol. Med., vol. 121, pp. 103805, Jun. 2020, doi: 10.1016/j.compbiomed.2020.103805.

[13] J. P. Cohen, et al., "Predicting covid-19 pneumonia severity on chest X-ray with deep learning," arXiv, vol. 12, no. 7, 2020, doi: 10.7759/cureus.9448.

[14] A. M. Ismael and A. Şengür, "Deep learning approaches for COVID-19 detection based on chest X-ray images," Expert Syst. Appl., vol. 164, p. 114054, Feb. 2021, doi: 10.1016/j.eswa.2020.114054.

[15] P. Afshar, S. Heidarian, F. Naderkhani, A. Oikonomou, K. N. Plataniotis, and A. Mohammadi, "COVID-CAPS: A capsule network-based framework for identification of COVID-19 cases from X-ray images," Pattern Recognition Letters. 2020, doi: 10.1016/j.patrec.2020.09.010.

[16] K. H. Shibly, S. K. Dey, M. T. U. Islam, and M. M. Rahman, "COVID faster R-CNN: A novel framework to diagnose novel Coronavirus Disease (COVID-19) in X-Ray images," Informatics Med. Unlocked, 2020, doi: 10.1016/j.imu.2020.100405.

[17] A. Makris, I. Kontopoulos, and K. Tserpes, "COVID-19 detection from chest X-ray images using deep learning and convolutional neural networks," 2020, doi: 10.1145/3411408.3411416.

[18] A. Jaiswal, N. Gianchandani, D. Singh, V. Kumar, and M. Kaur, "Classification of the COVID-19 infected patients using DenseNet201 based deep transfer learning," J. Biomol. Struct. Dyn., 2020, doi: 10.1080/07391102.2020.1788642.

[19] A. Narin, C. Kaya, and Z. Pamuk, "Automatic detection of coronavirus disease (COVID-19) using X-ray images and deep convolutional neural networks," arXiv. 2020.

[20] S. Q. Salih, H. K. Abdulla, Z. S. Ahmed, N. M. S. Surameery, and R. D. Rashid, "Modified AlexNet convolution neural network for Covid-19 detection using chest X-ray images," Kurdistan J. Appl. Res., 2020, doi: 10.24017/covid.14.

[21] P. Patel, "Chest X-ray (Covid-19 \& Pneumonia)," 2020. [Online]. Available. https://www.kaggle.com/prashant268/chestxray-covid19-pneumonia

[22] K. Simonyan and A. Zisserman, "VGG-16," arXiv Prepr., 2014

[23] C. Szegedy, V. Vanhoucke, S. Ioffe, J. Shlens, and Z. Wojna, "Rethinking the inception architecture for computer vision," Computer Vision and Pattern Recognition, 2016, doi: 10.1109/CVPR.2016.308.

[24] F. Chollet, "Xception: Deep learning with depthwise separable convolutions," IEEE Conference on Computer Vision and Pattern Recognition (CVPR), 2017, doi: 10.1109/CVPR.2017.195.

[25] G. Huang, Z. Liu, L. Van Der Maaten, and K. Q. Weinberger, "Densely connected convolutional networks," 2017, doi: 10.1109/CVPR.2017.243.

[26] C. Szegedy, S. Ioffe, V. Vanhoucke, and A. A. Alemi, "Inception-v4, inception-ResNet and the impact of residual connections on learning," Computer Vision and Pattern Recognition, 2017.

[27] M. Tan and Q. V. Le, "EfficientNet: Rethinking model scaling for convolutional neural networks," arXiv, 2019.

[28] A. I. Khan, J. L. Shah, and M. M. Bhat, "CoroNet: A deep neural network for detection and diagnosis of COVID19 from chest X-ray images," Comput. Methods Programs Biomed., vol. 196, 2020, doi: 10.1016/j.cmpb.2020.105581.

[29] A. Waheed, M. Goyal, D. Gupta, A. Khanna, F. Al-Turjman, and P. R. Pinheiro, "CovidGAN: Data Augmentation Using Auxiliary Classifier GAN for Improved Covid-19 Detection," IEEE Access, 2020, doi: 10.1109/ACCESS.2020.2994762. 